\title{
Breadboard Study of a Flexible High-Data Rate Transceiver Architecture (NNX16AD14G-Y2)
}

\author{
SHAYAN MOOKHERJEA \\ Department of Electrical and Computer Engineering, University of California, San Diego, MC 0407 La \\ Jolla CA 92093-0407 USA \\ Email:smookher@ucsd.edu
}

We experimentally study the breadboard implementation of an optical high-data rate transceiver architecture, which is based on an innovative design which generates a flexible number of communication streams from a single laser. Summary of a Project Outcomes report of research funded by NASA.

\section{Introduction and Goals}

The objective of this project is to develop the technology for silicon photonic transceiver components that can comprise a coherent laser optical communication and/or relay network in near-Earth space at wavelengths near $1550 \mathrm{~nm}$, for use on a satellite platform. Our proposed transceiver architecture generates a flexible number of communication streams from a single laser, which can be demultiplexed and individually modulated at the transmitter and decoded at the receiver. This scalability supports both high bit-rate communications with orders-of-magnitude reductions in size, weight, and energy consumption. Our strategy offers improvements on link performance and bandwidth compared to both RF communications and existing optical technologies.

\section{Activities}

The complete device consists of three building blocks: the recirculating variable-count frequency shifter (RVCFS), the high-order modulation format encoder, and the detector. The first is the most critical component. In keeping with the milestone schedule, we demonstrated the proof-of-concept verification of the RVCFS concept by assembling and operating a bread-board setup. We have successfully shown a variable number of tones (ranging from 1 to about 10) can be generated, and are using the measurement results to refine the realistic computational model[1].

The recirculating variable-count frequency shifter (RVCFS) component itself comprises of a single-sideband carrier-suppressed (SSBCS) modulator driven by an externally-supplied RF tone, a tunable optical bandpass filter, and (ideally) a gain section i.e., optical amplifier, which are placed in a loop. Our breadboard setup serves two purposes: (a) to validate the concept, and (b) to provide a benchmark for gradual insertion of microchip integrated versions of most of the components. 
Our setup is shown in Fig. 1, which has a few extra components (such as tap couplers for monitoring power levels at various points in the loop) and the electronics modules associated with some modules (e.g., SOA controller, filter controller), etc. A non-integrated (table-top) polarization-sensitive loop can be harder to stabilize than an integrated micro-chip version, and generous amounts of tape were used to hold fibers in place.

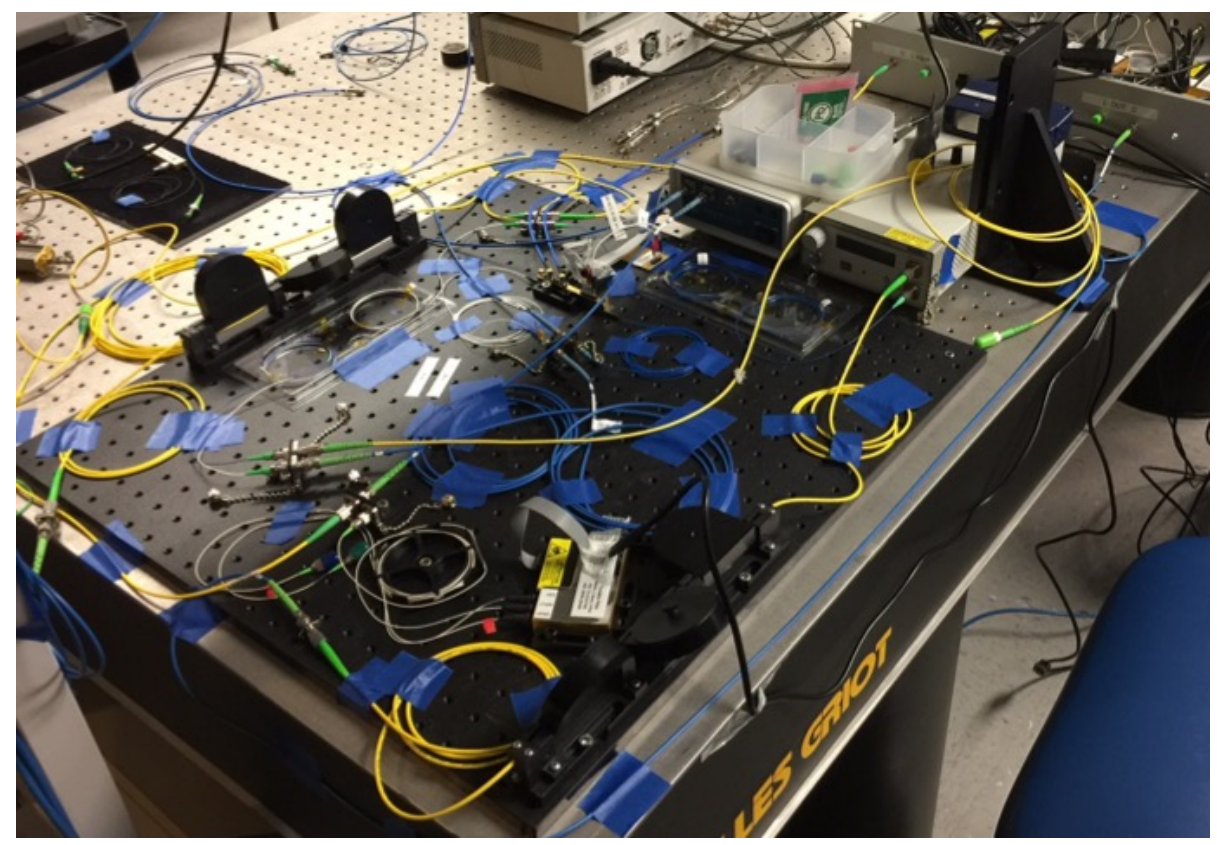

Figure 1 Photograph of breadboard setup for the recirculating loop generation of a variable number of carriers using discrete components and fiber interconnections.

Figure 2 shows a sequence of measurements of the optical spectrum with a controllable number of tones being generated from a single laser near $1550 \mathrm{~nm}$. The RF modulation frequency was chosen to be $10 \mathrm{GHz}$ in this example. The "control knob" over the number of tones is the center frequency of the optical loop filter.

The gain bandwidth of the SOA is much wider than the filter bandwidth, and is not the determining factor in the tone count. The optical filter is an important part of the RVCFS architecture. It is because of imperfect filtering that residual tones exist (at lower intensity levels) beyond the intended number of tones. Previously, we have studied optical wave propagation and bandpass filtering using the silicon coupled-resonator optical waveguide structure[2]-[6], which uses relatively low quality-factor resonators coupled in a serial cascade. However, such higher-order filtering devices are inherently sensitive to fabrication disorder and a simpler reduced-complexity structure may be optimal. A single-pole filter has an inherent tradeoff between the extinction ratio and the bandwidth; however, it is relatively easy to tune and control in 
practice and we have shown improvements in monitoring and stabilizing a high-Q silicon microresonator[7].

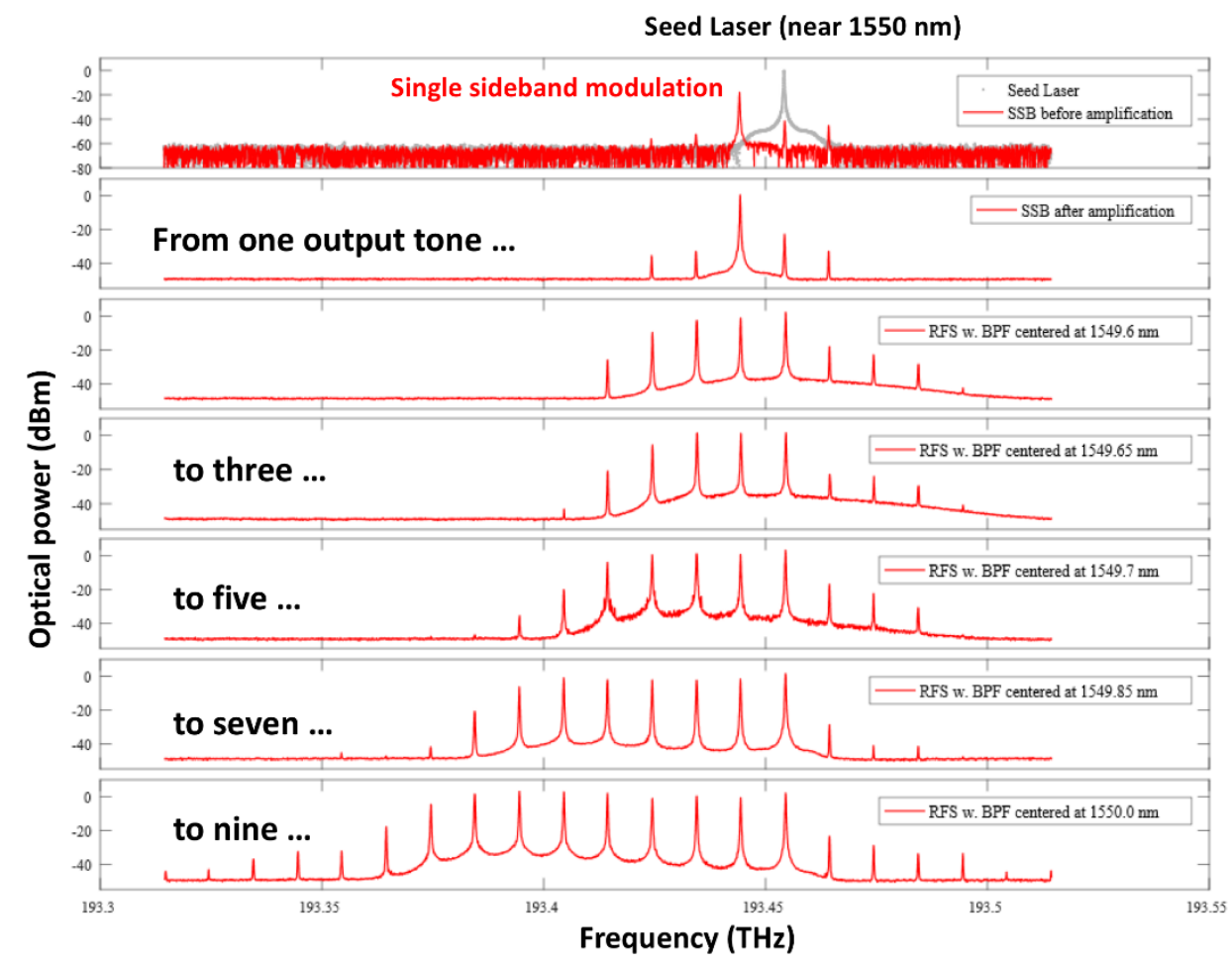

Figure 2 Experimental measurement of generating a variable number of carrier tones (spaced in this example by $10 \mathrm{GHz}$ ) using the recirculating loop approach. The control knob in this example is the center frequency of the bandpass filter which is part of the loop. The available loop bandwidth, and thus the number of generated tone, is dictated by the filter roll-off on the lower frequency side and the original seed laser tone on the higher frequency side.

\section{Impact}

Our research shows an innovative concept in wavelength-division multiplexing (WDM). The "control knob" over the number of tones is the center frequency of the optical loop filter, not the number of laser diodes fabricated on the chip. Compared to multiple lasers, the advantage of this approach to generating multiple tones are many-fold. We do not require multiple lasers but are able to implement WDM using a single laser. We enable more efficient power usage. We achieve granular (line by line) control over the number of carriers, which can enable user-defined dynamic communications rate adjustment over a very wide range.

This scheme also has an important fundamental advantage: in a communication system, the bit rate is reduced typically when the signal-to-noise ratio is low. Under those circumstances, channel capacity benefits from boosting the signal level (e.g., Shannon's channel capacity formula). This happens automatically in this scheme: when the filter bandwidth is narrow (i.e., fewer number of 
carriers, and lower overall bit rate), all the amplifier gain is dedicated to boosting the few circulating tones in the loop. This may help address the nearfar communications problem in space, where large variations in the distance between source and destination should be expected.

The components in the loop should have uniform performance across the bandwidth that spans all the tones, which could range from a few nanometers to several nanometers, depending on the RF frequency used to shift the tones in each pass through the SSB modulator. There are several couplers in the architecture which must be carefully designed, fabricated, and potentially trimmed after fabrication in the integrated chip. One of the advantages of the integrated device architecture is that directional couplers are well controlled, although the dispersion (i.e., wavelength sensitivity) of the directional coupler can be large. The same large dispersion issue also affects the coupled-resonator device and can sometimes be used for added functionality[8]. As an alternative, we can use multi-mode interference (MMI) couplers.

Since the single-sideband generation process results in tone-generation only to one side (here, the shorter frequency side) of the original carrier, we can achieve the same results as narrowing the filter bandwidth by moving the center frequency of the filter over towards higher frequencies (and not worrying about the filter passband width). This has the effect of compressing the available bandwidth for comb generation between the filter roll-off edge on the left and the carrier tone on the right. This itself results in a variable loop bandwidth, and this scheme is substantially easier (and lower insertion loss) than the technique of varying the optical filter bandwidth by programmable optical filters.

The work of one graduate student at UCSD was supported by this project. A self-funded masters student also contributed to research. The students received training in the fields of electrical engineering, physics, device fabrication and measurement. Results have been published in peer-reviewed journals and presented at internationally-recognized conferences, as well as workshops, seminars, and meetings.

\section{Potential Subsequent Extensions}

We use conventional silicon photonics technology for the single-sideband electro-optic modulator. However, an alternative emerging technology, which integrates thin-film lithium niobate with silicon photonic passive circuits, offers ultrahigh modulation bandwidth that is beyond the capabilities of traditional depletion-mode Mach-Zehnder modulators[9]. This may also help handle higher optical power, since silicon photonics devices suffer from two-photon absorption and free carrier absorption at higher intensities.

One of the challenges of high index contrast integrated photonics is the sensitivity to disorder. This is particularly true for the coupled-resonator configuration[10], [11] and affects the extinction ratio of filters. However, we 
have also studied ways to study and potentially compensate for disorder after fabrication [12]-[14].

All recirculating loop devices, especially those with gain in the loop, have to consider the effects of nonlinearity as the optical power is increased. Physics theory suggests that there are other interesting Kerr nonlinear effects that might occur in appropriately dispersion-engineered devices, such as ways to prevent the spreading of light through soliton generation [15]. However, these effects are mostly beyond the scope of this project. We have also not addressed how the optical gain block can be integrated on the same chip as the modulator and filter. Since the SSB modulator is polarization sensitive, it would be helpful to integrate the amplifier on the same chip so that the state of polarization does not drift or become misaligned.

\section{Open-Access Reporting Initiative}

PRAISE: This open-access document is provided in support of our PRAISE

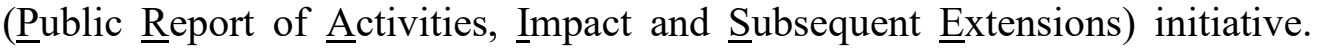
What is it? An open-access document shared with the public which describes the research outcomes of publicly-funded projects such as those funded by the U.S. NSF (National Science Foundation) and other agencies.

\section{References}

[1] X. Wang and S. Mookherjea, "Performance comparisons between semiconductor and fiber amplifier gain assistance in a recirculating frequency shifter," Opt. Lett., vol. 43, no. 5, p. 1011, Mar. 2018, doi: 10.1364/OL.43.001011.

[2] S. Mookherjea, "Spectral characteristics of coupled resonators," J. Opt. Soc. Am. B, vol. 23, no. 6, p. 1137, Jun. 2006, doi: 10.1364/JOSAB.23.001137.

[3] S. Mookherjea, D. S. Cohen, and A. Yariv, "Nonlinear dispersion in a coupled-resonator optical waveguide," Opt. Lett., vol. 27, no. 11, p. 933, Jun. 2002, doi: 10.1364/OL.27.000933.

[4] J. R. Ong and S. Mookherjea, "Quantum light generation on a silicon chip using waveguides and resonators," Opt. Express, vol. 21, no. 4, p. 5171, Feb. 2013, doi: 10.1364/OE.21.005171.

[5] M. L. Cooper et al., "235-ring Coupled-Resonator Optical Waveguides," in Conference on Lasers and Electro-Optics 2010, San Jose, California, 2010, p. CTuHH3. doi: 10.1364/CLEO.2010.CTuHH3.

[6] S. Mookherjea, J. R. Ong, X. Luo, and L. Guo-Qiang, "Electronic control of optical Anderson localization modes," Nature Nanotech, vol. 9, no. 5, pp. 365-371, May 2014, doi: 10.1038/nnano.2014.53.

[7] M. Savanier, R. Kumar, and S. Mookherjea, "Optimizing photon-pair generation electronically using a $p-i-n$ diode incorporated in a silicon 
microring resonator," Appl. Phys. Lett., vol. 107, no. 13, p. 131101, Sep. 2015, doi: 10.1063/1.4932047.

[8] S. Mookherjea, "Using gain to tune the dispersion relation of coupledresonator optical waveguides," IEEE Photon. Technol. Lett., vol. 18, no. 5, pp. 715-717, Mar. 2006, doi: 10.1109/LPT.2006.871144.

[9] X. Wang, P. O. Weigel, J. Zhao, M. Ruesing, and S. Mookherjea, "Achieving beyond-100-GHz large-signal modulation bandwidth in hybrid silicon photonics Mach Zehnder modulators using thin film lithium niobate," APL Photonics, vol. 4, no. 9, p. 096101, Sep. 2019, doi: 10.1063/1.5115243.

[10] M. L. Cooper and S. Mookherjea, "Modeling of Multiband Transmission in Long Silicon Coupled-Resonator Optical Waveguides," IEEE Photon. Technol. Lett., vol. 23, no. 13, pp. 872-874, Jul. 2011, doi: 10.1109/LPT.2011.2141657.

[11] S. Mookherjea and M. A. Schneider, "Avoiding bandwidth collapse in long chains of coupled optical microresonators," Opt. Lett., vol. 36, no. 23, p. 4557, Dec. 2011, doi: 10.1364/OL.36.004557.

[12] M. L. Cooper, G. Gupta, J. S. Park, M. A. Schneider, I. B. Divliansky, and S. Mookherjea, "Quantitative infrared imaging of silicon-on-insulator microring resonators," Opt. Lett., vol. 35, no. 5, p. 784, Mar. 2010, doi: 10.1364/OL.35.000784.

[13] S. Mookherjea and H. R. Grant, "High dynamic range microscope infrared imaging of silicon nanophotonic devices," Opt. Lett., vol. 37, no. 22, p. 4705, Nov. 2012, doi: 10.1364/OL.37.004705.

[14] Y. Shen, I. B. Divliansky, D. N. Basov, and S. Mookherjea, "Perfect set-and-forget alignment of silicon photonic resonators and interferometers," in Optical Fiber Communication Conference/National Fiber Optic Engineers Conference 2011, Los Angeles, California, 2011, p. PDPC3. doi: 10.1364/OFC.2011.PDPC3.

[15] B. Crosignani, A. Yariv, and S. Mookherjea, "Nonparaxial spatial solitons and propagation-invariant pattern solutions in optical Kerr media," Opt. Lett., vol. 29, no. 11, p. 1254, Jun. 2004, doi: 10.1364/OL.29.001254. 